\title{
Preclinical study of cinobufagin as a promising anti-colorectal cancer agent
}

\author{
Xing-sheng Lu ${ }^{1,2, *}$, Yin-biao Qiao ${ }^{3, *}, \mathrm{Ya} \mathrm{Li}^{4, *}$, Bo Yang ${ }^{3}$, Min-bin Chen ${ }^{5}$, Chun-gen \\ Xing ${ }^{1}$ \\ ${ }^{1}$ Department of General Surgery, The Second Affiliated Hospital of Soochow University, Suzhou, China \\ ${ }^{2}$ Department of General Surgery, Suzhou Municipal Hospital, Suzhou, China \\ ${ }^{3}$ Department of Hepatobiliary Surgery, The Third Hospital Affiliated to Soochow University, Changzhou City, Jiangsu, China \\ ${ }^{4}$ Institute of Neuroscience, Soochow University, Suzhou, China \\ ${ }^{5}$ Department of Medical Oncology, Kunshan First People's Hospital Affiliated to Jiangsu University, Kunshan, China \\ * Co-first authors \\ Correspondence to: Chun-gen Xing, email: profxingsz9@163.com \\ Min-bin Chen, email: cmb1981@163.com
}

Keywords: colorectal cancer (CRC), cinobufagin (CBG), ER stress, mTOR and apoptosis

Received: October 15, 2016

Accepted: October 28, 2016

Published: November 23, 2016

\section{ABSTRACT}

Here, we assessed the anti-colorectal cancer (CRC) cell activity of cinobufagin (CBG). We found that CBG exerted potent cytotoxic and anti-proliferative activity against CRC lines (HCT-116 and HT-29) and primary human CRC cells. Meanwhile, it activated apoptosis, and disrupted cell-cycle progression in the cells. At the signaling level, CBG treatment in CRC cells provoked endoplasmic reticulum stress (ER stress), the latter was evidenced by caspase-12 activation, CHOP expression, as well as PERK and IRE1 phosphorylations. Contrarily, the ER stress inhibitor salubrinal, the caspase-12 inhibitor and CHOP shRNA remarkably attenuated CBG-induced CRC cell death and apoptosis. Further, CBG in-activated mammalian target or rapamycin complex 1 (mTORC1), which appeared responsible for proliferation inhibition in CRC cells. Introduction of a constitutively-active S6K1 ("ca-S6K1") restored proliferation of CBG-treated CRC cells. Finally, CBG intraperitoneal injection suppressed HCT116 xenograft tumor growth in the nude mice. CHOP upregulation and mTORC1 in-activation were also noticed in CBG-treated HCT-116 tumors. The results of this preclinical study suggest that CBG could be tested as promising anti-CRC agent.

\section{INTRODUCTION}

Colorectal cancer (CRC) is a major threat to human health, and it ranks one of leading causes of cancerrelated mortalities in the world [1-3]. Conventional chemotherapies aren't able to kill CRC cells with preexisting and/or acquired resistances [4]. Therefore, our group [5-10] and others are focusing on exploring novel and more potent anti-CRC agents [11]. Treatment cancer cells with Traditional Chinese Medicine (TCM) has been a research focus for many years [12]. One of these TCM, Chansu, is extracted from parotoid glands of the Chinese toad [13]. In ancient China and other Asian countries, Chansu has been widely utilized for the treatment of inflammation, anaesthesia and arrhythmia [13]. Cinobufagin $(\mathrm{CBG})$ is a primary and active component from Chansu [14]. Recent preclinical studies have tested its anti-cancer activity [15-17]. However, its potential effect in CRC cells has not been extensively studied. More importantly, the underlying signaling mechanisms of CBG-mediated cancer cell killing effect are largely unknown.

Endoplasmic reticulum (ER) is the organelle that is key the synthesis, post-translational modification, proper folding, and maturation of new proteins $[18,19]$. Interruption normal ER functions, i.e. by anti-cancer drugs, could result in pathological ER stress [18, 19], causing several unfolded protein responses (UPR) [20]. ER stress could lead to up-regulation of ER chaperones, including pro-apoptotic C/EBP homologous protein (CHOP) [21] and many others. Several ER membrane receptors, including double-stranded RNA-activated 
protein kinase (PKR)-like ER kinase (PERK), activating transcription factor 6 (ATF6) and inositol-requiring enzyme 1 (IRE1), could act as the sensors of ER stress [22]. In the current study, we provided evidences to show that CBG-induced CRC cell death is associated with ER stress activation.

\section{RESULTS}

\section{Cinobufagin (CBG) exerts potent cytotoxic and anti-proliferative activity against human CRC cells}

To study the potential effect of $\mathrm{CBG}$ on $\mathrm{CRC}$ cells, HCT-116 CRC cells [6] were cultured in complete medium, and were treated with designated concentrations (1-250 ng/mL) of CBG. MTT cell viability assay results demonstrated that $\mathrm{CBG}$ dose-dependently inhibited HCT-116 cell survival (Figure 1A). CBG's IC50, the concentration that inhibited $50 \%$ of HCT-116 cell survival, was less than $50 \mathrm{ng} / \mathrm{mL}$ at 48 and 72 hours (Figure 1A). The lowest concentration of CBG $(1 \mathrm{ng} / \mathrm{mL})$ failed to inhibit HCT-116 cell survival (Figure 1A). Further, CBG also displayed a time-dependent response in inhibiting HCT-116 cells (Figure 1A). As early as 24 hours after
CBG (50-250 ng/mL) treatment, a significant viability reduction was noticed, and it was more dramatic at 48 and 72 hours (Figure 1A). Notably, CBG (100 ng/mL, 48 hours) was also cytotoxic to HT-29 CRC cells (Figure 1B). Clonogenicity assay results demonstrated that $\mathrm{CBG}$ effectively decreased the number of viable colonies of HCT-116 cells (Figure 1C) and HT-29 cells (Figure 1D), further confirming its cytotoxicity against CRC cells. As shown in Figure $1 \mathrm{E}$ and $1 \mathrm{~F}, \mathrm{CBG}$ was also antiproliferative when added to HCT-116 cells (Figure 1E, a dose-dependent response was observed) and HT-29 cells (Figure 1F). The BrdU OD was decreased in CBG-treated CRC cells (Figure 1E and 1F). Notably, BrdU OD was normalized to the cell viability (MTT OD) to exclude the influence of cell death (Figure 1E and F, same for all the BrdU assays of the study).

The effect of CBG on other cancer cells was also analyzed. As shown in Figure $1 \mathrm{G}$, in two primary human colon cancer cell lines ("Pri Colon-1/-2"), treatment with CBG (100 ng/mL, 48 hours) also significantly decreased cell survival. Meanwhile, same CBG treatment was also cytotoxic to PANC-1 pancreatic cancer cells (Figure 1G) [23]. Intriguingly, the CBG treatment $(100 \mathrm{ng} / \mathrm{mL}, 48$ hours) was somehow non-cytotoxic to the primary colon epithelial cancer cells ("Colon Epi") and to the HPDE6c7

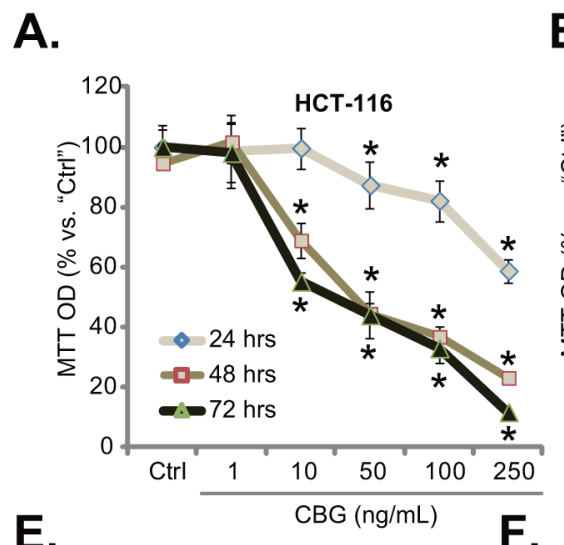

B. C

D.

E.

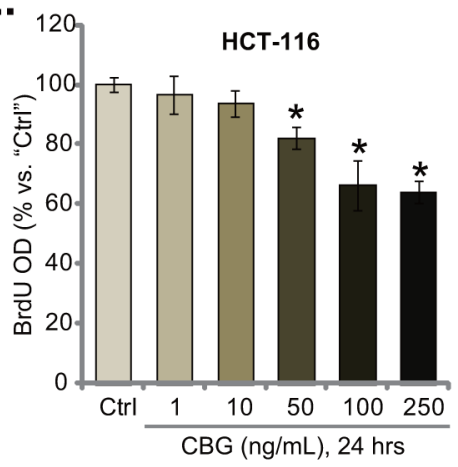

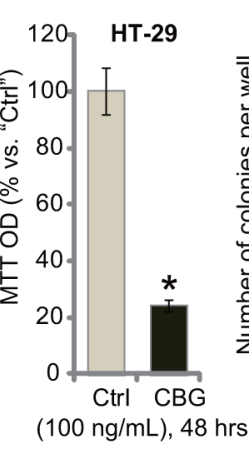

G.

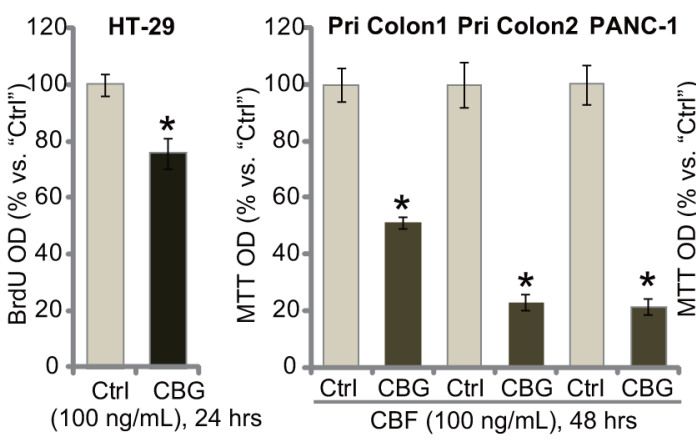

$\mathrm{H}$.
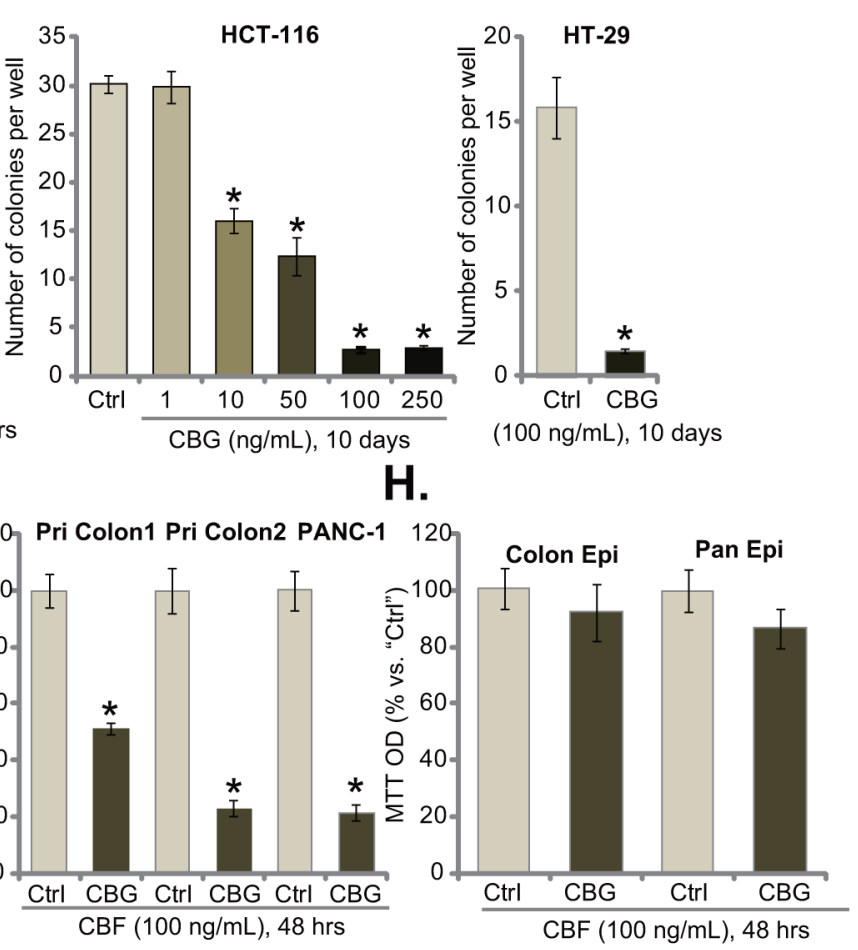

Figure 1: Cinobufagin (CBG) exerts potent cytotoxic and anti-proliferative activity against human CRC cells. Listed cancer cells or non-cancerous epithelial cells were treated with/out designated concentrations of cinobufagin (CBG, 1-250 ng/mL), cells were further cultured for indicated time; Cell survival was tested by MTT assay A, B, G and H. and clonogenicity assay $\mathbf{C}$ and D.; Cell proliferation was tested by the BrdU ELISA assay $\mathbf{E}$ and $\mathbf{F}$. Experiments in this figure were repeated five times, with similar results obtained. n=5 for each repeat. "Ctrl” stands for untreated control group (Same for all figures). * $\boldsymbol{p}<0.05$ vs. group of "Ctrl". 
pancreatic epithelial cells ("Pan Epi") (Figure 1H), these results indicated a selective cytotoxicity of $\mathrm{CBG}$ to cancerous cells.

\section{Cinobufagin (CBG) provokes apoptosis in CRC cells}

Next, we tested CBG's effect on CRC cell apoptosis, which was tested by previously described apoptosis assays [5-10]. TUNEL staining assay (Figure 2A and 2B), Histone-DNA ELISA assay (Figure 2C and 2D) and Annexin V FACS assay (Figure 2E and 2F) results demonstrated that CBG, at tested concentrations (10-250 $\mathrm{ng} / \mathrm{mL}$ ) efficiently provoked apoptosis in both HCT-116 cells and HT-29 cells (Figure 2A-2F). The TUNELpositive cells (Figure 2A and 2B), the apoptosis ELISA OD (Figure 2C and 2D) and Annexin V percentage (Figure $2 \mathrm{E}$ and $2 \mathrm{~F}$ ) were all increased significantly following $\mathrm{CBG}$ (10-250 ng/mL) treatment in CRC cells. Further, TUNEL assay results showed that CBG (100 ng/mL) activated apoptosis in two lines of primary colon cancer cells ("Pri Colon-1/-2") as well as in PANC-1 pancreatic cancer cells (Figure 2G). On the other hand, no profound apoptosis was observed in CBG (100 ng/mL)-treated colon epithelial cells ("Colon Epi") and HPDE6c7 pancreatic epithelial cells ("Pan Epi") (Figure 2H). These results clearly show that $\mathrm{CBG}$ provokes apoptosis in $\mathrm{CRC}$ cells.

\section{Cinobufagin (CBG) disturbs CRC cell cycle progression}

The effect of CBG on CRC cell cycle progression was also tested. As shown in Figure 3A, following treatment of CBG (100 ng/mL, 24 hours) in HCT-116 cells, the percentage of G1 phase cells was significantly decreased. Correspondingly, the percentages of $\mathrm{S}$ and G2-M phase cells were increased (Figure 3A). Quantified results in Figure 3B further demonstrated G2-M arrest in CBG-treated HCT-116 cells (Figure 3B). Same experiments were also performed in PANC-1 cells, and similar G2-M arrest was noticed (Figure 3C and 3D). These results demonstrate that $\mathrm{CBG}$ induces $\mathrm{G} 2-\mathrm{M}$ arrest in the cancer cells.

\section{CBG-mediated CRC cell death is associated with ER stress activation}

When analyzing potential caspases that were possibly activated by $\mathrm{CBG}$, we found that caspase- 12 activity was significantly increased in CBG-treated HCT-116 cells and
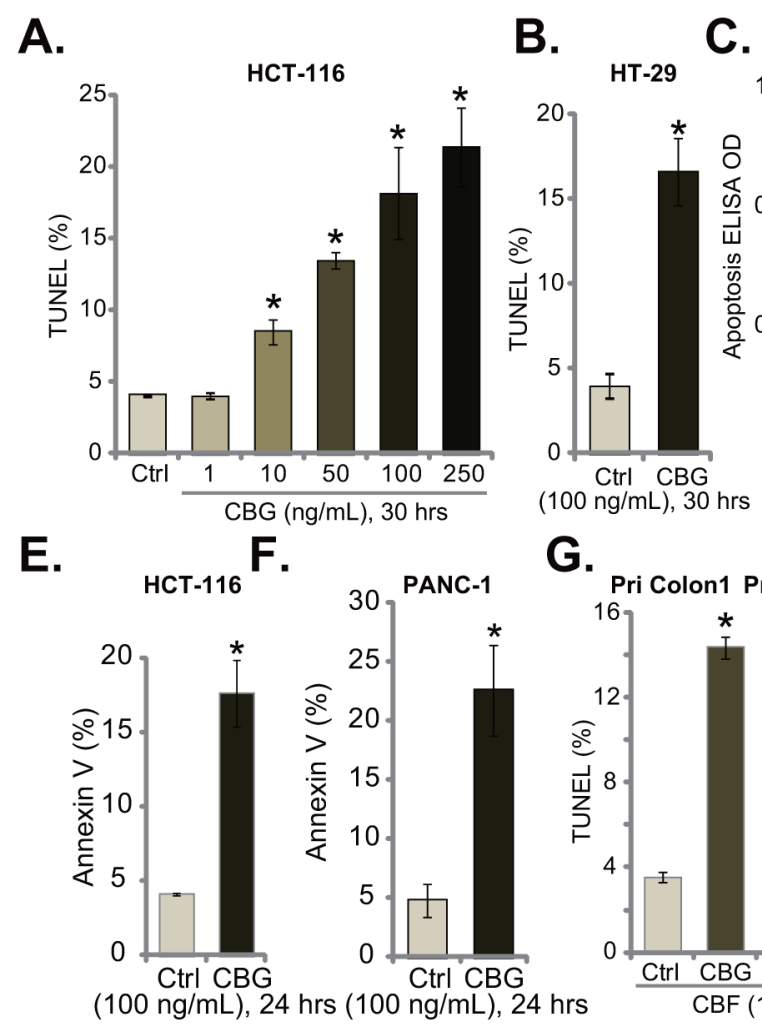

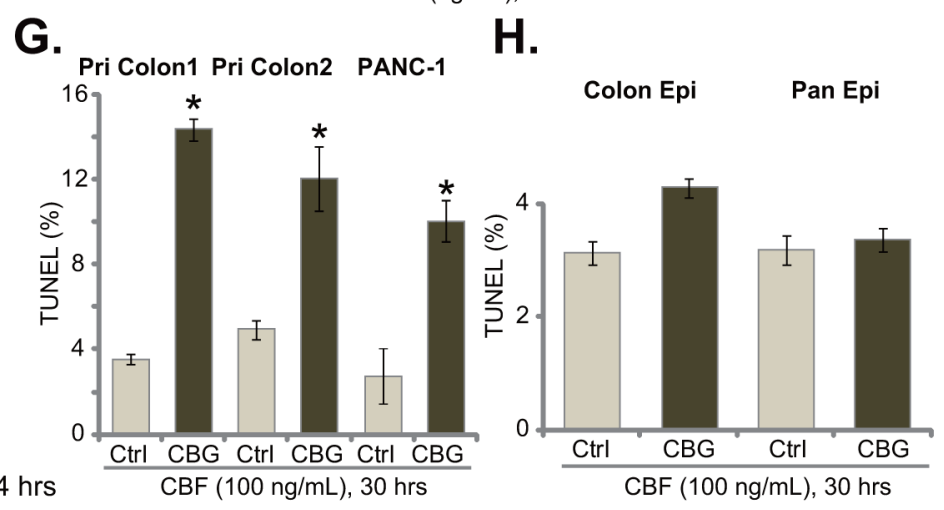

D.
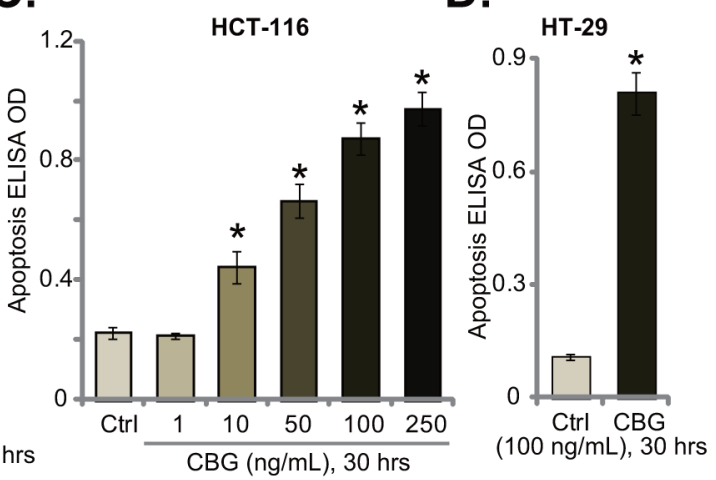

H.

Figure 2: Cinobufagin (CBG) provokes apoptosis in CRC cells. Listed cancer cells or non-cancerous epithelial cells were treated with/out designated concentrations of cinobufagin (CBG, 1-250 ng/mL), cells were further cultured for indicated time; Cell apoptosis was tested by TUNEL staining assay A, B, G and H., histone-DNA ELISA assay $\mathbf{C}$ and D. and Annexin V FACS assay (E and F., quantified results were presented at the right panels). Experiments in this figure were repeated three times, with similar results obtained. ${ }^{*} \boldsymbol{p}<0.05$ vs. group of "Ctrl". 
HT-29 cells (Figure 4A and 4B). Activation of caspase-12 is a characteristic marker of ER stress apoptosis pathway $[24,25]$, we thus tested other ER stress-associated proteins in CBG-treated cells. Western blot results demonstrated that treatment of HCT-116 cells (Figure 4C) and HT29 cells (Figure 4D) with CBG (10-250 ng/ml) induced CHOP expression, PERK and IRE1 phosphorylations, indicating ER stress activation [18, 20]. Remarkably, the ER stress inhibitor salubrinal [26], the caspase-12 inhibitor zATADfmk as well as the pan caspase inhibitor zVADfmk largely inhibited CBG (100 ng/ml)-induced HCT-116 cell death (Figure 4E) and apoptosis (Figure 4F), indicating the requirement of ER stress in mediating $\mathrm{CBG}$ 's cytotoxicity.

To further support our hypothesis, shRNA strategy was applied to knockdown CHOP, a key protein of ER stress apoptosis pathway [21]. The three CHOP shRNAs with non-overlapping sequences were applied, and each of them efficiently downregulated CHOP in CBG-treated HCT-116 cells (Figure 4G). Remarkably, CHOP shRNA knockdown largely attenuated CBG's killing of HCT-116 cells (Figure $4 \mathrm{H}$ and $4 \mathrm{I}$ ). Thus, CHOP and ER stress activation mediated CBG-induced CRC cell death and apoptosis.

\section{CBG inhibits mTORC1 activation in CRC cells}

Interestingly, we showed that the ER stress inhibitor salubrinal and the caspase inhibitors (zATADfmk and
zVADfmk) failed to reverse or attenuate cell proliferation inhibition by $\mathrm{CBG}$ in HCT-116 cells (Figure 5A). Similarly, CHOP shRNA knockdown in HCT-116 cells also didn't ameliorate CBG-induced proliferation inhibition (Figure 5B). These results indicated that ER stress apparently didn't participate in CBG-induced anti-proliferative action. Therefore, we tested several proliferation-associated signalings in CBG-treated cancer cells. Significantly, we found that CBG (50/100 ng/mL) treatment in HCT-116 cells largely inhibited mammalian target of rapamycin (mTOR) complex 1 (mTORC1) activation, the latter was evidenced by phosphorylations ("p") of S6 and 4EBP1 [27, 28] (Figure 5C). Similar results were also obtained in PANC-1 cells, where CBG (50/100 ng/mL) largely inhibited S6 and 4EBP1 phosphorylations (Figure 5D).

To study the link between CBG-induced mTORC1 in-activation and proliferation inhibition, we introduced the constitutively-active S6K1 (T389E, "ca-S6K1") [29] into HCT-116 cells, and two stably lines ("caS6K1-L1/2") were established. Quantified results in Figure 5E demonstrated that ca-S6K1 restored S6 and S6K1 phosphorylations in CBG (100 ng/mL)-treated HCT-116 cells to almost control level. Consequently, cell proliferation was almost recovered by ca-S6K1 in HCT-116 cells (Figure 5F). Similarly in PANC-1 cells, introduction ca-S6K1 restored cell proliferation even

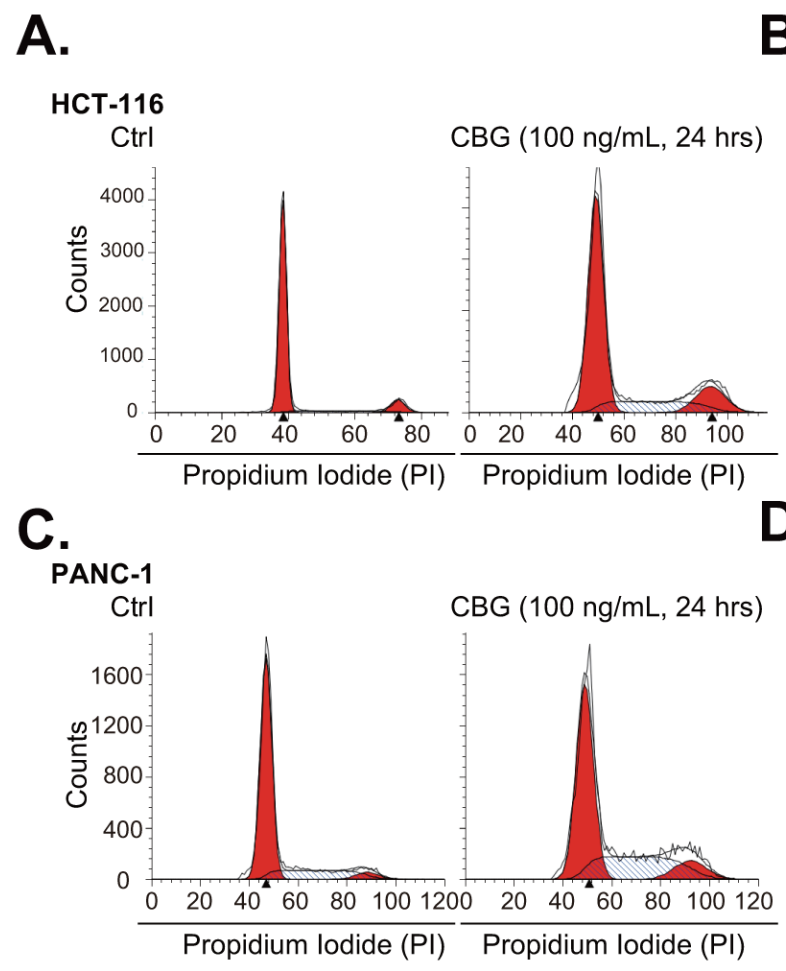

B.

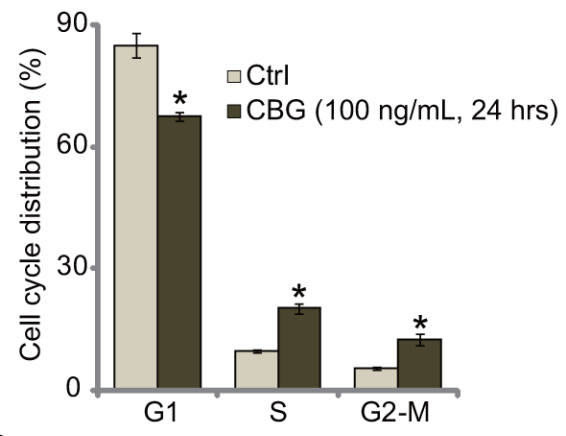

D.

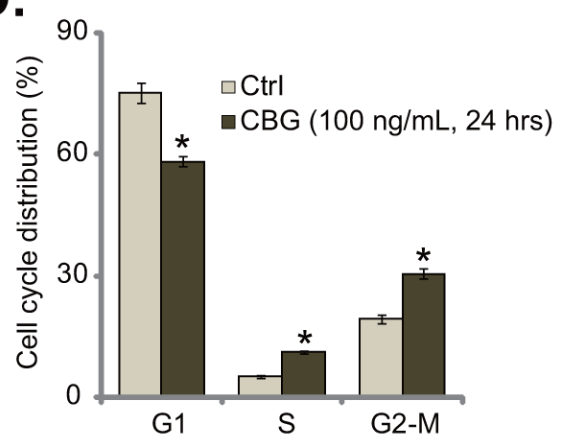

Figure 3: Cinobufagin (CBG) disturbs CRC cell cycle progression.HCT-116 cells and PANC-1 cells were treated with/out cinobufagin (CBG, $100 \mathrm{ng} / \mathrm{mL})$, cells were further cultured for 24 hours; Cell cycle distribution was shown $\mathbf{A}$ and $\mathbf{C}$.; Data of three set repeats were quantified $\mathbf{B}$ and $\mathbf{D} . * \boldsymbol{p}<0.05$ vs. group of "Ctrl". 
with CBG (100 ng/mL) treatment (Figure 5G). Notably, ca-S6K1 alone also promoted proliferation of above cancer cells (Figure 5F and 5G), further confirming the positive role of mTOR in promoting cell proliferation. These results suggest that CBG-mediated proliferation inhibition in above cancer cells could be due to mTORC1 in-activation.

\section{Cinobufagin (CBG) inhibits HCT-116 tumor growth in nude mice}

To study the anti-cancer activity by $\mathrm{CBG}$ in vivo, the mouse xenograft tumor model was established. A significant number of HCT-116 cells were injected in the flanks of nude mice. Within three weeks, xenograft HCT-

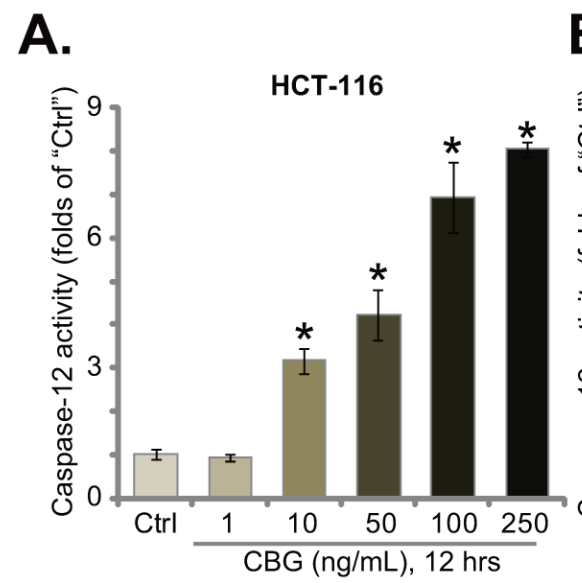

B. C.

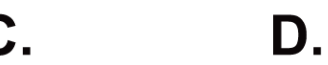

HT-29 HCT-116 HT-29
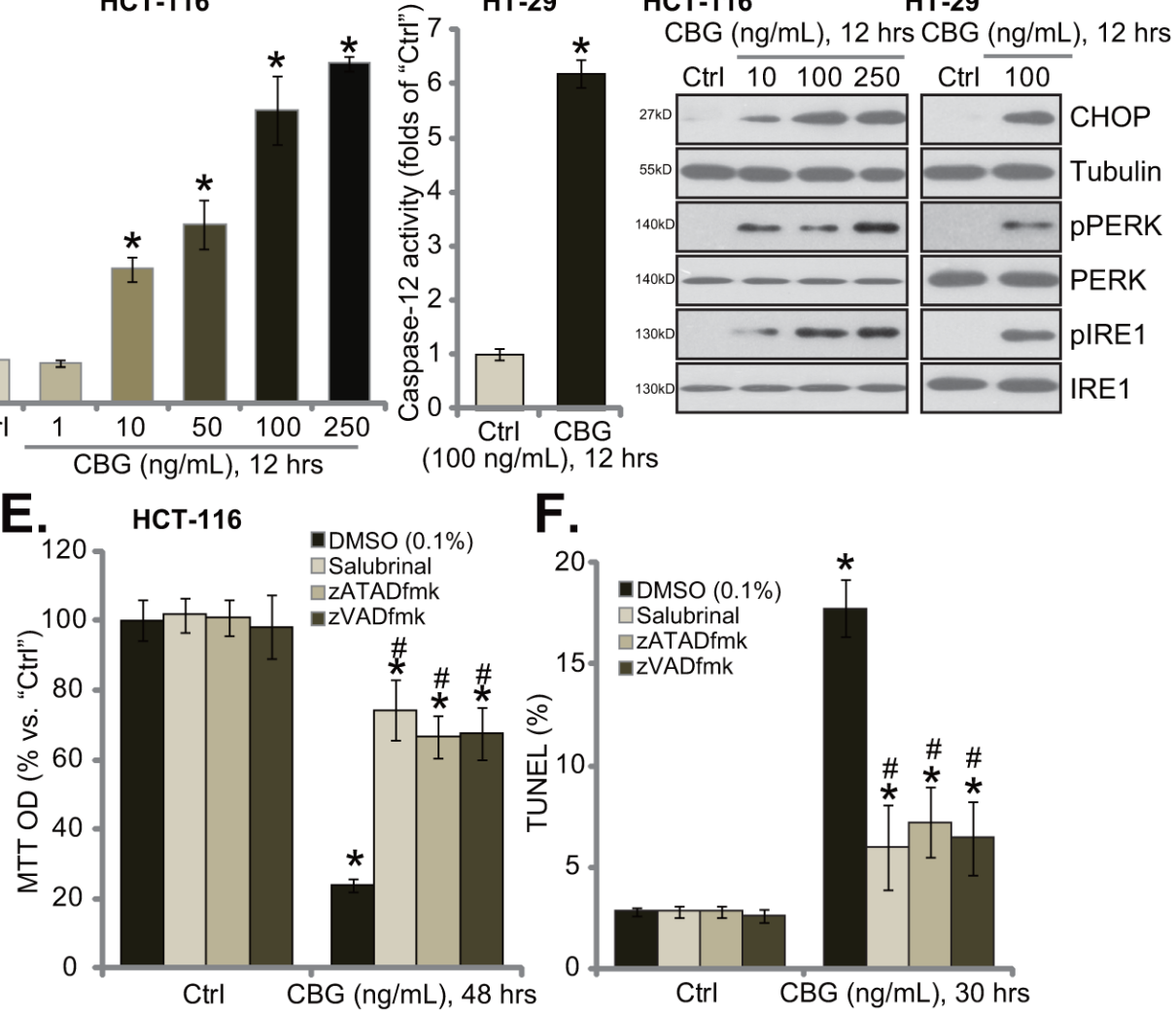

$\mathbf{F}$.

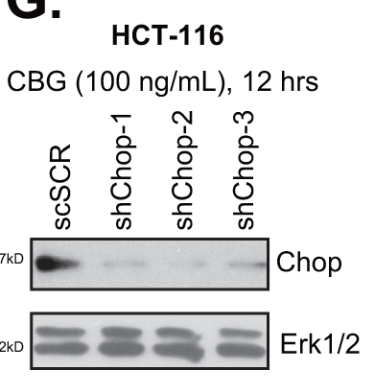

H.

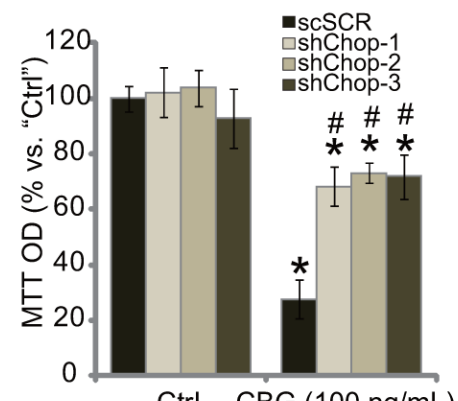

I.

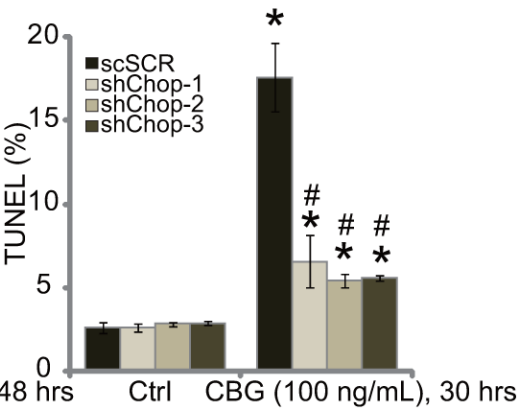

Figure 4: CBG-mediated CRC cell death is associated with ER stress activation. Listed cancer cells were treated with/ out designated concentrations of cinobufagin (CBG, 1-250 ng/mL), cells were further cultured for indicated time; Caspase-12 activity $\mathbf{A}$ and B. and expressions of listed ER stress proteins $\mathbf{C}$ and D. were tested. HCT-116 cells were pre-treated 1 hour with salubrinal (10 $\mu \mathrm{M})$, zATADfmk $(50 \mu \mathrm{M})$, or zVADfmk $(50 \mu \mathrm{M})$, followed by cinobufagin $(\mathrm{CBG}, 100 \mathrm{ng} / \mathrm{mL})$ stimulation, cells were further cultured for indicated time; Cell viability (E., MTT assay) and apoptosis (F., TUNEL staining assay) were tested. Stably HCT-116 with scrambleshRNA ("shSCR") or CHOP-shRNA ("shCHOP-1/-2/-3") were treated with cinobufagin (CBG, $100 \mathrm{ng} / \mathrm{mL}$ ) for indicated time; CHOP expression G., cell survival H. and apoptosis I. were tested. Experiments in this figure were repeated four times, with similar results obtained. * $\boldsymbol{p}<0.05$ vs. group of "Ctrl". " $\boldsymbol{p}<0.05$ vs. CBG treatment group of "DMSO" (E and F) or "shSCR" (H and I). 
116 tumors were established. The growth curve results in Figure 6A demonstrated that CBG administration (10 mg/ kg body weight, i.p., daily) significantly inhibited HCT116 tumor growth in the nude mice. The tumor volumes in CBG-treated mice were significantly lower than those of vehicle control mice (Figure 6A). Yet, the mice body weights in the CBG mice were not significantly different from that of vehicle control mice (Figure 6B), indicating the relative safety of the $\mathrm{CBG}$ regimen here. Notably, no apparent toxicities were noticed in the CBG-treated mice. Next, we wanted to know if the signaling changes by CBG in vitro were also achieved in vivo. At day three following initial CBG treatment, HCT-116 tumors were isolated, and expressions of above signaling proteins in tumor lysates were tested by Western blot assay. Quantified results in Figure 6C clearly demonstrated CHOP upregulation and p4EBP1 inhibition in HCT-116 tumors with CBG administration. Therefore, in line with the in vitro findings,
A.

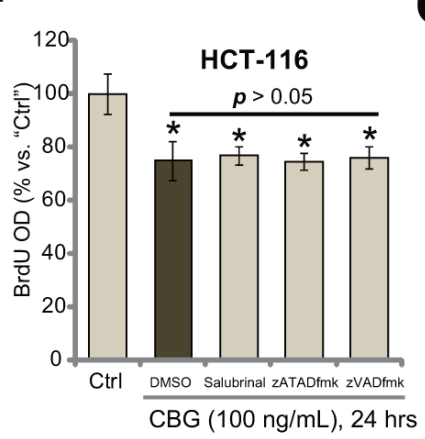

B.

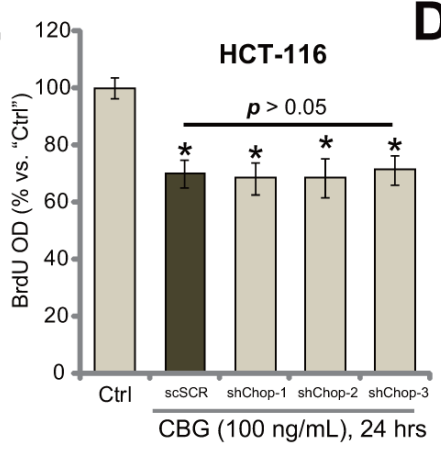

C.

\section{HCT-116}

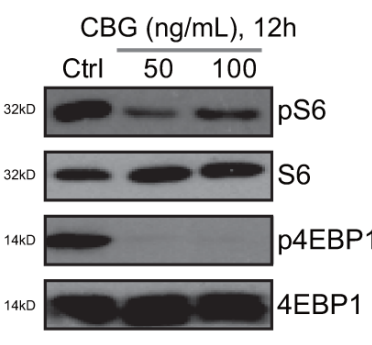

D.

\section{PANC-1}

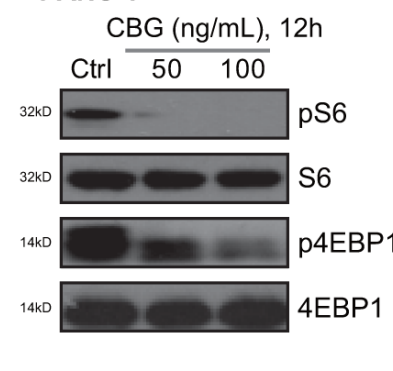

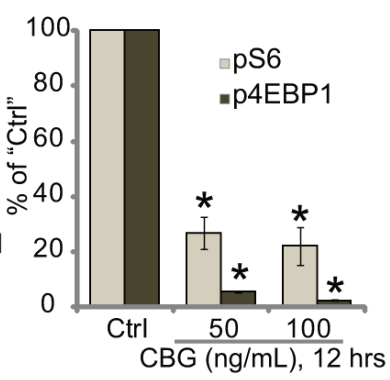

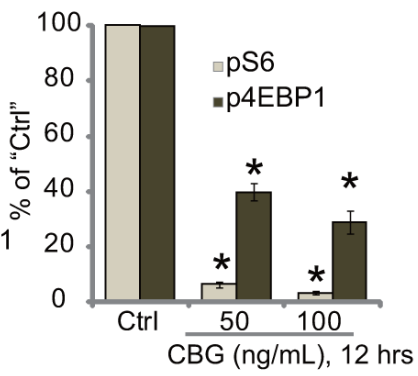

E.

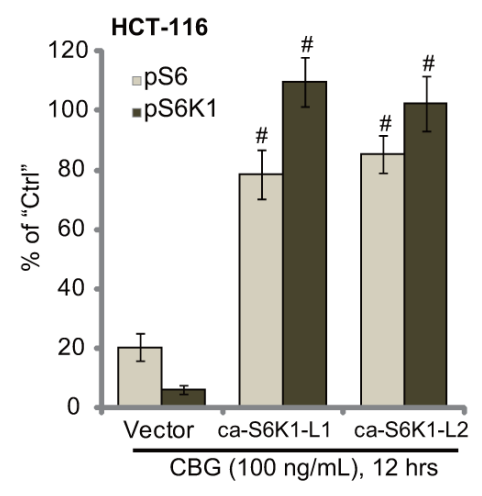

$F$.

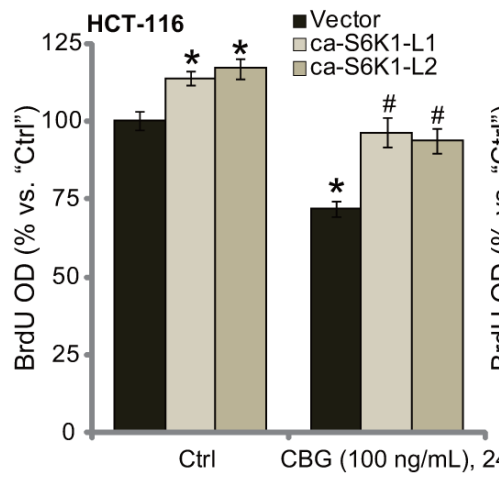

G.

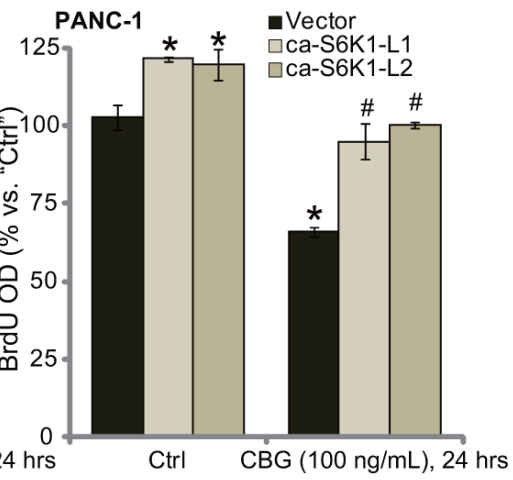

Figure 5: CBG inhibits mTORC1 activation in CRC cells. HCT-116 cells were pre-treated 1 hour with salubrinal (10 $\mu \mathrm{M})$, zATADfmk $(50 \mu \mathrm{M})$, or zVADfmk $(50 \mu \mathrm{M})$, followed by cinobufagin $(\mathrm{CBG}, 100 \mathrm{ng} / \mathrm{mL})$ stimulation for applied time; Cell proliferation was tested by BrdU ELISA assay A. Stably HCT-116 with scramble-shRNA ("shSCR") or CHOP-shRNA ("shCHOP-1/-2/-3") were treated with cinobufagin $(\mathrm{CBG}, 100 \mathrm{ng} / \mathrm{mL})$ for indicated time; Cell proliferation was tested $\mathbf{B}$. HCT-116 cells or PANC-1 cells were treated with/out cinobufagin $(\mathrm{CBG}, 50 / 100 \mathrm{ng} / \mathrm{mL})$ for 12 hours, expressions of listed proteins were shown (C and D., data of four repeats were quantified at right panels). Stably HCT-116 cells E and F. or PANC-1 cells G., expressing empty vector ("pGCL-flag-puro") or the constitutively-active S6K1 (T389E, "ca-S6K1", two different lines "L1/L2"), were treated with/out cinobufagin (CBG, 100 ng/mL) for indicated time; Relative S6 and S6K1 phosphorylations (\% vs. "Ctrl”" cells) were shown (E, integration of three repeats); Cell proliferation was tested by BrdU ELISA assay (F and G). Experiments in this figure were repeated four times, with similar results obtained. $* \boldsymbol{p}<0.05$ vs. group of "Ctrl". " $\boldsymbol{p}<0.05$ vs. "CBG" treatment group of "Vector" cells (E-G). 
CBG administration in vivo possibly also induced ER stress activation and mTORC1 in-activation in HCT-116 tumors.

\section{DISCUSSION}

In the current study, we showed that CBG exerted cytotoxic, pro-apoptotic and anti-proliferative actions against established (HCT-116 and HT-29) and primary CRC cells. In vivo, CBG administration largely inhibited HCT-116 tumor growth in nude mice. ER stress activation and $\mathrm{mTORC} 1$ in-activation apparently mediated CBG's actions in CRC cells. Interestingly, ER stress inhibition, by adding salubrinal, zATADfmk, or CHOP shRNAs, didn't totally abolish CBG-mediated CRC cell death (Figure 4). Therefore, other mechanisms besides ER stress should also participate in CBG-induced killing of CRC cells. For example, it has been shown that $\mathrm{CBG}$ exerted cytotoxicity in multiple myeloma cells via activating ROS-mediated
MAPK signaling pathway [30]. Qi et al., showed that CBG activated Fas- and mitochondria-mediated pathways to kill hepatocellular carcinoma cells [16]. The study by $\mathrm{Yu}$ et al., suggested a pivotal role of p53 activation in mediating CBG-induced prostate cancer cell apoptosis [17]. Thus, it will be interesting to test the association between ER stress activation and these other cascades in CBG-treated CRC cells.

Although ER stress activation mediated CBGinduced CRC cell death, blockage of this pathway didn't bring back proliferation of CRC cells. Here, we suggested that mTORC1 inactivation could be important for CBG's anti-proliferative activity in CRC cells. Remarkably, introduction of the ca-S6K1 restored proliferation in CBG-treated CRC cells. Notably, there was no crosstalk between ER stress activation and mTORC1 in-activation in CBG-treated cells. Salubrinal, zATADfmk, or CHOP shRNAs had no effect on CBG-induced mTORC1 inactivation in CRC cells; Meanwhile, the ca-S6K1 also
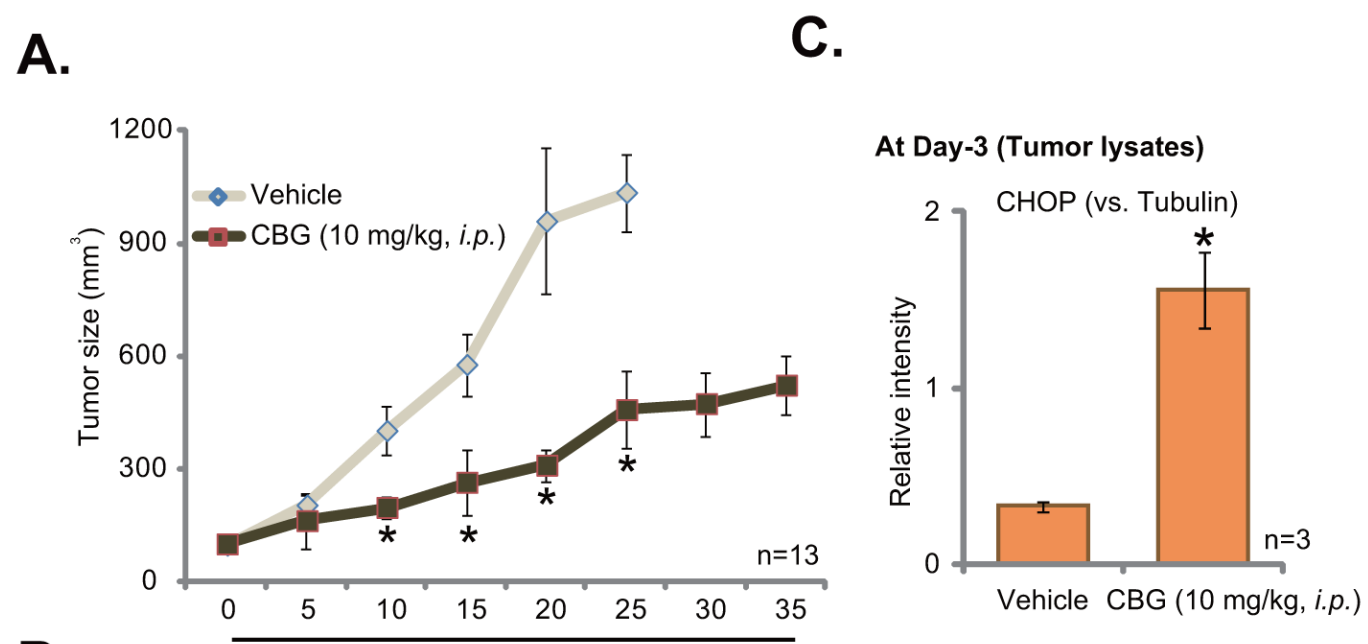

B.

Days post CBG administration

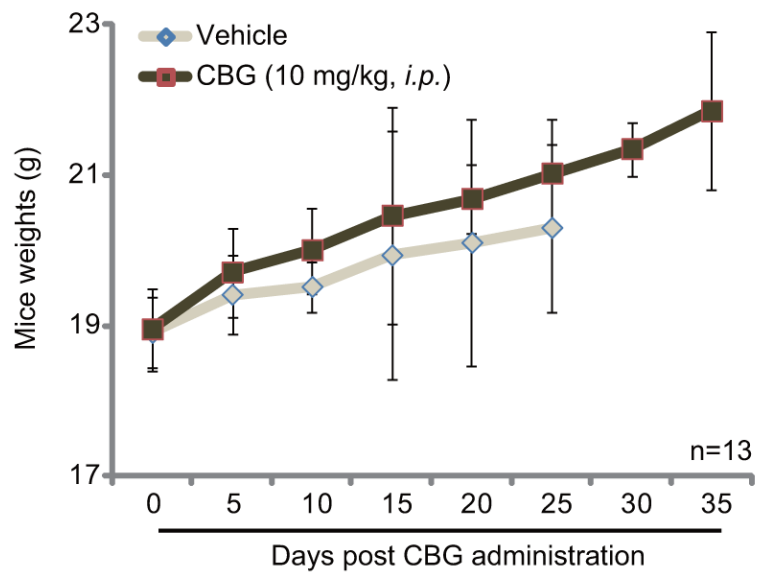

At Day-3 (Tumor lysates)

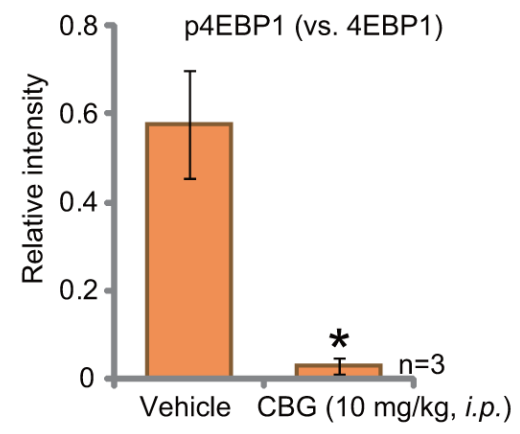

Figure 6: Cinobufagin (CBG) inhibits HCT-116 tumor growth in nude mice. HCT-116 tumor-bearing nude mice ( $\mathrm{n}=13$ per group) were administrated with cinobufagin (CBG, $10 \mathrm{mg} / \mathrm{kg}$, i.p., daily) or vehicle control ("Vehicle") for 20 consecutive days, tumor volumes (in $\mathrm{mm}^{3}$ ) A. and mice body weights (B., in grams) were recorded every 5 days. At day three after initial CBG administration, three HCT-116 tumors per group were isolated, expressions of listed proteins in tumor lysates were tested by Western blot assay, and data were integrated and quantified C.; $* \boldsymbol{p}<0.05$ vs. group of "vehicle". 
failed to inhibit ER stress activation by CBG. Therefore, these two signalings by $\mathrm{CBG}$ appeared parallel to each other, and exerted separated functions in CRC cells.

Intriguingly, we showed that CBG treatment failed to induce significant cytotoxicity to normal colon and pancreatic epithelial cells. Thus, CBG treatment was relatively safe to the normal cells. One possibility could be that mTORC1 (p4EBP1/pS6) was only hyper-activated in cancer cells, but was quite low in the epithelial cells. Another possibility is that CBG-induced ER stress activation somehow could only promote cancerous cell death, as ER function is often hyper-activated in cancer cells [20,31]. Normal epithelial cells could then be tolerate to ER stress by CBG.

One important finding of this study is that $\mathrm{CBG}$ was also cytotoxic when added to PANC-1 pancreatic cancer cells, where ER stress activation and mTORC1 in-activation were similarly noticed. Therefore, it would be interesting to test CBG's anti-cancer activity against other solid tumor cells, and whether the mentioned two signalings (ER stress activation and mTORC1 inactivation) could also been observed in other cancer cells. Together, these preclinical results suggest that $\mathrm{CBG}$ could be a promising chemotherapeutic agent for $\mathrm{CRC}$ and possible other cancers.

\section{MATERIALS AND METHODS}

\section{Chemicals and reagents}

Cinobufagin $(\mathrm{CBG})$ and the ER stress inhibitor salubrinal [26] were purchased from Sigma Chemicals (St. Louis, MO). The pan-caspase inhibitor (z-VAD-fmk) and the specific caspase-12 inhibitor (z-ATAD-fmk) [32] were purchased from Calbiochem (Darmstadt, Germany). All phosphorylation antibodies and their non-phosphorylation control antibodies were purchased from Cell Signaling Technology (Beverly, MA). All other antibodies were obtained from Santa Cruz Biotech (Santa Cruz, CA). The concentrations of agents applied and the treatment durations were chosen based on published literatures and results from our pre-experiments.

\section{Culture of established cell lines}

As reported early [5-10], CRC lines HT-29 and HCT-116 were maintained in DMEM medium with $10 \%$ FBS, Penicillin/Streptomycin and $4 \mathrm{mM}$ L-glutamine, in a $\mathrm{CO}_{2}$ incubator at $37^{\circ} \mathrm{C}$. PANC-1 pancreatic cancer cells, purchased from the Cell Bank of Shanghai Institute of Biological Science (Shanghai, China), were cultured in above DMEM medium. HPDE6c7, an immortalized pancreatic epithelial cell line [33], was purchased from GuangZhou Jennio Biotech (Guangzhou, China). HPDE6c7 cells were cultivated in DMEM supplemented with $10 \%$ FBS. For all the cell lines, DNA fingerprinting and profiling were performed every 6 months to confirm the origin of the cell line, and to distinguish the cell line from cross-contamination. All cell lines were subjected to mycoplasma and microbial contamination examination. Population doubling time, colony forming efficiency, and morphology under phase contrast were also measured every 6 months under defined conditions to confirm the phonotype of cell line.

\section{Primary culture of colon cancer and epithelial cells}

As previously described [8, 10], the fresh colon cancer tissues along with the surrounding normal epithelial tissues were separated carefully under the microscope. Tissues were thoroughly washed and minced, which were then mechanically dissociated and filtered via a $70 \mu \mathrm{m}$ strainer. Single-cell suspensions were achieved by re-suspending cells in $0.15 \%(\mathrm{w} / \mathrm{v})$ collagenase I (Sigma) containing medium. Cells were then cultured in the medium as described [10]. The study was approved by the institutional review board (IRB) of all authors' institutions. Procedures were conducted according to the principles expressed in the Declaration of Helsinki. Two male patients $(55 / 61$ year old $)$ with primary colon cancer were enrolled. Written-informed consent was obtained from each participant.

\section{Methyl thiazol tetrazolium (MTT) assay}

Cell viability was assessed by the MTT assay as described [7, 8, 34].

\section{Colony formation assay}

As previously reported $[8,35]$, cells with applied treatment were suspended in agar-containing medium, which were then added on the top of a culture dish. After 10 days of incubation, the number of colonies were fixed, stained and manually counted.

\section{Annexin V FACS assay of cell apoptosis}

After treatment, cells were washed and incubated with Annexin V-FITC (3 $\mu \mathrm{g} / \mathrm{mL}$, Invitrogen, Shanghai, China) and Binding Buffer (Invitrogen). A total of 10,000 cells of each sample were analyzed by flow cytometry in a FACS (Beckton Dickinson, Cytoflex, Shanghai, China). The percentage of Annexin $\mathrm{V}$ was utilized as a quantitative measurement of cell apoptosis.

\section{Histone-DNA Enzyme-linked immunosorbent assay (ELISA) assay}

As previously described [8], cell apoptosis was quantified by Histone-DNA ELISA PLUS kit (Roche Applied Science, Shanghai, China). 


\section{Western blot assay}

Western blot assay was performed as previously described [5-10]. Blot intensity was quantified by ImageJ software $(\mathrm{NIH})$.

\section{Caspase-12 activity assay}

Following treatment of cells, cytosolic extracts ( $20 \mu \mathrm{g}$ per treatment) were added to the caspase assay buffer [36] plus the caspase-12 substrate ATAD-7-AFC (Invitrogen). The amount of liberated AFC was tested by a spectrofluorometer (Thermo-Fisher, Shanghai, China).

\section{TUNEL staining assay}

TUNEL (Terminal deoxynucleotidyl transferase dUTP nick end labeling) In Situ Cell Death Detection Kit (Roche, Shanghai, China) was applied to evaluate cell apoptosis. TUNEL percentage (TUNEL/DAPI $\times 100 \%$ ) was calculated from at least 100 cells per treatment.

\section{BrdU incorporation assay}

Cells $\left(0.25 \times 10^{5}\right.$ cells/well $)$ were seeded in complete medium. The BrdU ELISA colorimetric assay kit (Cell Signaling) was applied to test cell proliferation [37].

\section{CHOP shRNA knockdown}

Three lentiviral shRNAs targeting non-overlapping sequence of human $C H O P$ sequence were designed, synthesized and validated by Genepharm Co. (Shanghai, China). Ten $\mu \mathrm{L} / \mathrm{mL}$ of lentiviral shRNA was added to the HCT-116 cells for 48 hours; Stable HCT-116 clones expressing targeted-shRNA were selected by puromycin $(5.0 \mu \mathrm{g} / \mathrm{mL})$ for $4-6$ passages. CHOP expression in the resistant colonies was detected by Western blot assay.

\section{The constitutively active S6K1 construct and transfection}

The constitutively active S6K1 (T389E, "ca-S6K1flag-puro") and the empty vector (pGCL-flag-puro) were gifts from Dr. Chen's group [29]. The construct was transfected via Lipofectamine 2000 reagents (Invitrogen) [29]. Cells were subjected to puromycin $(5.0 \mu \mathrm{g} / \mathrm{mL})$ selection for 4-6 passages. "ca-S6K1" expression in the stable cells was again verified by Western blot assay.

\section{Xenograft assay}

Five million HCT116 cells in PBS $(200 \mu \mathrm{L})$ were subcutaneously injected into each mouse. Within three weeks, the xenografted tumors were established with the size around $100 \mathrm{~mm}^{3}$. The nude mice (aged 7-8 weeks, weighting 18-19 g) were equally divided into two groups: intraperitoneal (i.p.) injection and control. CBG was initially dissolved in ethanol and was then diluted in 10 $\%$ propylene glycol solution. The daily dose given to i.p. group was $10 \mathrm{mg} / \mathrm{kg}$ of body weight, while an equal amount of injection solution without $\mathrm{CBG}$ was given as the vehicle control. Mice were sacrificed when the tumor grew over $100 \mathrm{~mm}^{3}$. Tumor volumes were recorded, calculated via the following formula: $\pi / 6 \times$ larger diameter $\times($ smaller diameter $)^{2}$. All studies were performed in accordance with the standards of ethical treatment approved by the Institutional Animal Care and Use Committee (IACUC).

\section{Statistical analysis}

Data were presented as mean \pm standard deviation (SD). Statistics were analyzed by one-way ANOVA using the SPSS 18.0 software (SPSS Inc., Chicago, IL). Significance was chosen as $\boldsymbol{p}<0.05$.

\section{ACKNOWLEDGMENTS}

This work was generously supported by Suzhou Key Medical Center (LCZX201505), Suzhou Science and Technology Development Program (SZS201618) and National Natural Science Foundation of China (81672970), and the Second Affiliated Hospital of Soochow University Preponderant Clinical Discipline Group Project Funding.

\section{CONFLICTS OF INTEREST}

The authors have no conflict of interests.

\section{Author contributions}

All authors carried out the experiments, participated in the design of the study and performed the statistical analysis, conceived of the study, and participated in its design and coordination and helped to draft the manuscript. All authors read and approved the final manuscript.

\section{REFERENCES}

1. Schmoll HJ and Stein A. Colorectal cancer in 2013: Towards improved drugs, combinations and patient selection. Nat Rev Clin Oncol. 2014; 11:79-80.

2. Kuipers EJ, Rosch T and Bretthauer M. Colorectal cancer screening--optimizing current strategies and new directions. Nat Rev Clin Oncol. 2013; 10:130-142.

3. Brouquet A and Nordlinger B. Metastatic colorectal cancer outcome and fatty liver disease. Nat Rev Gastroenterol Hepatol. 2013; 10:266-267. 
4. Liscovitch M and Lavie Y. Cancer multidrug resistance: a review of recent drug discovery research. IDrugs. 2002; 5:349-355.

5. Chen MB, Yang L, Lu PH, Fu XL, Zhang Y, Zhu YQ and Tian Y. MicroRNA-101 down-regulates sphingosine kinase 1 in colorectal cancer cells. Biochem Biophys Res Commun. 2015; 463:954-960.

6. Chen MB, Jiang Q, Liu YY, Zhang Y, He BS, Wei MX, $\mathrm{Lu}$ JW, Ji Y and Lu PH. C6 ceramide dramatically increases vincristine sensitivity both in vivo and in vitro, involving AMP-activated protein kinase-p53 signaling. Carcinogenesis. 2015; 36:1061-1070.

7. Chen MB, Wei MX, Han JY, Wu XY, Li C, Wang J, Shen $\mathrm{W}$ and $\mathrm{Lu}$ PH. MicroRNA-451 regulates AMPK/mTORC1 signaling and fascin1 expression in HT-29 colorectal cancer. Cell Signal. 2014; 26:102-109.

8. Chen MB, Zhang Y, Wei MX, Shen W, Wu XY, Yao C and $\mathrm{Lu} \mathrm{PH}$. Activation of AMP-activated protein kinase (AMPK) mediates plumbagin-induced apoptosis and growth inhibition in cultured human colon cancer cells. Cell Signal. 2013; 25:1993-2002.

9. Chen MB, Wu XY, Tao GQ, Liu CY, Chen J, Wang LQ and Lu PH. Perifosine sensitizes curcumin-induced anticolorectal cancer effects by targeting multiple signaling pathways both in vivo and in vitro. Int J Cancer. 2012; 131:2487-2498.

10. Li C, Cui JF, Chen MB, Liu CY, Liu F, Zhang QD, Zou $\mathrm{J}$ and $\mathrm{Lu} \mathrm{PH}$. The preclinical evaluation of the dual mTORC1/2 inhibitor INK-128 as a potential anti-colorectal cancer agent. Cancer Biol Ther. 2015; 16:34-42.

11. Palta M, Czito BG and Willett CG. Colorectal cancer: adjuvant chemotherapy for rectal cancer-an unresolved issue. Nat Rev Clin Oncol. 2014; 11:182-184.

12. Wang S, Penchala S, Prabhu S, Wang J and Huang Y. Molecular basis of traditional Chinese medicine in cancer chemoprevention. Curr Drug Discov Technol. 2010; 7:67-75.

13. Hong Z, Chan K and Yeung HW. Simultaneous determination of bufadienolides in the traditional Chinese medicine preparation, liu-shen-wan, by liquid chromatography. J Pharm Pharmacol. 1992; 44:1023-1026.

14. Yang Z, Luo H, Wang $\mathrm{H}$ and Hou H. Preparative isolation of bufalin and cinobufagin from Chinese traditional medicine ChanSu. J Chromatogr Sci. 2008; 46:81-85.

15. Zhang G, Wang C, Sun M, Li J, Wang B, Jin C, Hua P, Song G, Zhang Y, Nguyen LL, Cui R, Liu R, Wang L and Zhang $\mathrm{X}$. Cinobufagin inhibits tumor growth by inducing intrinsic apoptosis through AKT signaling pathway in human nonsmall cell lung cancer cells. Oncotarget. 2016; 7:28935-28946. doi: 10.18632/oncotarget.7898.

16. Qi F, Inagaki Y, Gao B, Cui X, Xu H, Kokudo N, Li A and Tang W. Bufalin and cinobufagin induce apoptosis of human hepatocellular carcinoma cells via Fas- and mitochondriamediated pathways. Cancer Sci. 2011; 102:951-958.
17. Yu CH, Kan SF, Pu HF, Jea Chien E and Wang PS. Apoptotic signaling in bufalin- and cinobufagin-treated androgen-dependent and -independent human prostate cancer cells. Cancer Sci. 2008; 99:2467-2476.

18. Healy SJ, Gorman AM, Mousavi-Shafaei P, Gupta S and Samali A. Targeting the endoplasmic reticulum-stress response as an anticancer strategy. Eur J Pharmacol. 2009; 625:234-246.

19. Wlodkowic D, Skommer J, McGuinness D, Hillier C and Darzynkiewicz Z. ER-Golgi network--a future target for anti-cancer therapy. Leuk Res. 2009; 33:1440-1447.

20. Verfaillie T, Garg AD and Agostinis P. Targeting ER stress induced apoptosis and inflammation in cancer. Cancer Lett. 2013; 332:249-264.

21. Oyadomari S and Mori M. Roles of CHOP/GADD153 in endoplasmic reticulum stress. Cell Death Differ. 2004; 11:381-389.

22. Hetz $\mathrm{C}$. The unfolded protein response: controlling cell fate decisions under ER stress and beyond. Nat Rev Mol Cell Biol. 2012; 13:89-102.

23. Gong L, Yang B, Xu M, Cheng B, Tang X, Zheng P, Jing $\mathrm{Y}$ and $\mathrm{Wu}$ GJ. Bortezomib-induced apoptosis in cultured pancreatic cancer cells is associated with ceramide production. Cancer Chemother Pharmacol. 2014; 73:69-77.

24. Yoneda T, Imaizumi K, Oono K, Yui D, Gomi F, Katayama $\mathrm{T}$ and Tohyama M. Activation of caspase-12, an endoplastic reticulum (ER) resident caspase, through tumor necrosis factor receptor-associated factor 2-dependent mechanism in response to the ER stress. J Biol Chem. 2001; 276:13935-13940.

25. Nakagawa T, Zhu H, Morishima N, Li E, Xu J, Yankner BA and Yuan J. Caspase-12 mediates endoplasmic-reticulumspecific apoptosis and cytotoxicity by amyloid-beta. Nature. 2000; 403:98-103.

26. Boyce M, Bryant KF, Jousse C, Long K, Harding HP, Scheuner D, Kaufman RJ, Ma D, Coen DM, Ron D and Yuan J. A selective inhibitor of eIF2alpha dephosphorylation protects cells from ER stress. Science. 2005; 307:935-939.

27. Dancey J. mTOR signaling and drug development in cancer. Nat Rev Clin Oncol. 2010; 7:209-219.

28. Sabatini DM. mTOR and cancer: insights into a complex relationship. Nat Rev Cancer. 2006; 6:729-734.

29. Xin Y, Shen XD, Cheng L, Hong DF and Chen B. Perifosine inhibits S6K1-Glil signaling and enhances gemcitabine-induced anti-pancreatic cancer efficiency. Cancer Chemother Pharmacol. 2014; 73:711-719.

30. Baek SH, Kim C, Lee JH, Nam D, Lee J, Lee SG, Chung WS, Jang HJ, Kim SH and Ahn KS. Cinobufagin exerts anti-proliferative and pro-apoptotic effects through the modulation ROS-mediated MAPKs signaling pathway. Immunopharmacol Immunotoxicol. 2015; 37:265-273.

31. Wang M and Kaufman RJ. The impact of the endoplasmic reticulum protein-folding environment on cancer development. Nat Rev Cancer. 2014; 14:581-597. 
32. Zhu YR, Xu Y, Fang JF, Zhou F, Deng XW and Zhang YQ. Bufotalin-induced apoptosis in osteoblastoma cells is associated with endoplasmic reticulum stress activation. Biochem Biophys Res Commun. 2014; 451:112-118.

33. Bu HQ, Liu DL, Wei WT, Chen L, Huang H, Li Y and Cui JH. Oridonin induces apoptosis in SW1990 pancreatic cancer cells via p53- and caspase-dependent induction of p38 MAPK. Oncol Rep. 2014; 31:975-982.

34. Chen MB, Shen WX, Yang Y, Wu XY, Gu JH and Lu PH. Activation of AMP-activated protein kinase is involved in vincristine-induced cell apoptosis in B16 melanoma cell. J Cell Physiol. 2010; 226:1915-1925.
35. Chen MB, Yang L, Lu PH, Fu XL, Zhang Y, Zhu YQ and Tian Y. MicroRNA-101 down-regulates sphingosine kinase 1 in colorectal cancer cells. Biochem Biophys Res Commun. 2015.

36. Wu K, Li N, Sun H, Xu T, Jin F and Nie J. Endoplasmic reticulum stress activation mediates Ginseng Rg3-induced anti-gallbladder cancer cell activity. Biochem Biophys Res Commun. 2015; 466:369-375.

37. Zhang YM, Zhang ZQ, Liu YY, Zhou X, Shi XH, Jiang Q, Fan DL and Cao C. Requirement of Galphai1/3-Gab1 signaling complex for keratinocyte growth factor-induced PI3K-AKT-mTORC1 activation. J Invest Dermatol. 2015; 135:181-191. 